\title{
Envejecimiento activo en Bizkaia: situación comparada en el contexto europeo
}

\section{Yolanda González-Rábago}

Departamento de Sociología 2, Universidad del País Vasco (UPV/EHU)

Grupo de Investigación en Determinantes Sociales de la Salud y

Cambio Demográfico (OPIK)

$<$ yolanda.gonzalezr@ehu.eus>

\section{Unai Martín}

Departamento de Sociología 2, Universidad del País Vasco (UPV/EHU) Grupo de Investigación en Determinantes Sociales de la Salud y Cambio Demográfico (OPIK)

\section{Amaia Bacigalupe}

Departamento de Sociología 2, Universidad del País Vasco (UPV/EHU) Grupo de Investigación en Determinantes Sociales de la Salud y

Cambio Demográfico (OPIK)

\section{Sergio Murillo}

Dirección General de Promoción de la Autonomía Personal, Diputación Foral de Bizkaia

\begin{abstract}
Biztanleriaren zahartze prozesua, mendebaldeko gizarteetan gero eta garrantzitsuagoa den fenomenoa dugu, batez ere, Europako testuinguruan. Horren ondorioz, 'zahartze aktiboa' sustatzera doazen politika publikoak jarri dira abian, gero eta ugariagoa den populazio heldu horren egoera eta gizarte ondorioak hobesteko asmoarekin. Active Ageing Index (AAI) deiturikoa, Europar herrialdeen arteko populazio helduaren zahartze aktiboaren maila konparatzeko tresna da. Bizkaiko Foru Aldundia Europatik datorren lan ildo honekin lerrokatu da, bere politika publikoak monitorizatzeko populazio helduaren zahartze aktiboaren diagnositik abiatzea, alegia. Artikuluaren helburua da Bizkaiko 55 urte eta gehiagokoen zahartze aktiboaren egoera AAl-aren bitartez analizatu, gainontzeko Europako herrialdeekin konparatu, eta zahartze aktiboak Bizkaian izan ditzakeen mugak eta potentzialtasunak identifikatzea.
\end{abstract}

\section{GAKO-HITZAK:}

Zahartze aktiboa, Bizkaia, adierazleak, politika publikoak.
El envejecimiento demográfico es un fenómeno de creciente importancia en las sociedades occidentales, especialmente en Europa. Por ello, se han puesto en marcha políticas públicas enmarcadas en el denominado 'envejecimiento activo' que pretenden mejorar la situación y consecuencias sociales de una cada vez más numerosa población mayor. El Îndice de Envejecimiento Activo es un instrumento que mide el envejecimiento activo y ofrece un diagnóstico comparado a escala europea de la situación de la población mayor. La Diputación Foral de Bizkaia se ha sumado a esta línea de trabajo utilizando el índice para monitorizar sus políticas públicas en esta materia. El objetivo de este artículo es analizar la situación de envejecimiento activo de la población de $\mathbf{5 5}$ y más años en Bizkaia a través del índice, de forma comparada con el resto de países de la Unión Europea, identificando carencias y potencialidades en el desarrollo de un envejecimiento activo.

\section{Palabras Clave:}

Envejecimiento activo, Bizkaia, indicadores, políticas públicas. 


\section{Introducción}

\subsection{El envejecimiento de la población en Bizkaia como reto social y político}

El envejecimiento de la población implica un proceso de cambio de la estructura de edades de una sociedad, con un incremento relativo de la proporción de las personas mayores. Este proceso se deriva principalmente de dos causas fundamentales: por un lado, el aumento de la esperanza de vida, que provoca que un mayor número de personas llegue a edades avanzadas; $y$, por otro lado, el descenso de la natalidad, que disminuye la población infantil y juvenil, y aumenta el peso de las personas de edad avanzada.

Este incremento de las personas mayores ha tenido especial importancia en las sociedades europeas. En 2013, la población de 65 y más años representaba en la Unión Europea (UE-28) el 18,4 \% del total, y las proyecciones demográficas indican que esta proporción se incrementará fuertemente durante los próximos años, llegando al $24,1 \%$ en 2030 y el $28,2 \%$ en 2050 (Comisión Europea, 2015). En los países del sur de Europa, el proceso de envejecimiento demográfico ha sido más rápido y el peso de la población mayor es en la actualidad más alto que en el resto de la Unión Europea, alcanzando el $\mathbf{2 1 , 2} \%$ en Italia y el $20,3 \%$ en Grecia (ibídem). El Estado español se sitúa un poco por debajo de la media europea, con un $17,9 \%$ de población mayor de 64 años. Sin embargo, dentro del Estado se dan diferencias relevantes, siendo las comunidades autónomas del norte las que se encuentran más envejecidas. Dentro de éstas, destaca la Comunidad Autónoma de País Vasco (CAPV), con un mayor porcentaje de personas mayores $(20,7 \%)$, fruto tanto de una muy baja fecundidad durante las últimas décadas del siglo pasado como de una esperanza de vida mayor que la media del Estado (Luxán y Martín Roncero, 2012).

En Bizkaia, la población mayor de 64 años ascendía en 2014 a 242.905 personas, lo que suponía el $21,2 \%$ del total (Eustat, 2014a). La proporción de población mayor es más numerosa entre las mujeres, 142.418 personas ( $24 \%$ del total), que entre los hombres, 100.487 personas (18,2 \% del total). Además, esta diferencia se va ampliando a medida que aumenta la edad. De hecho, la población sobreenvejecida, es decir, de 80 años o más, que asciende a 19.775 personas, estaba compuesta mayoritariamente por mujeres (74\%). Este proceso de envejecimiento, que lleva produciéndose con especial intensidad desde los años noventa del siglo XX, está lejos de finalizar, y las proyecciones demográficas indican que en 2025 el $42 \%$ de la población en Bizkaia tendrá 55 años 0 más (Eustat, 2014b).

El envejecimiento de la población constituye uno de los mayores logros sociales de la época reciente, ya que conlleva una "democratización de la supervivencia” (Pérez Díaz, 2006), por la cual la mayor parte de personas consigue vivir hasta edades que, en épocas anteriores, sólo alcanzaban partes muy reducidas de la población. El fenómeno conlleva importantes procesos de transformación social, y más allá del uso interesado que algunos sectores económicos y financieros han realizado del envejecimiento demográfico, sí parece cierto que este cambio en la estructura de edades de la población supone y supondrá en el futuro uno de los mayores retos políticos y sociales.

Es por ello por lo que los Gobiernos, tanto nacionales, regionales como locales, están comenzando a reflexionar en torno al envejecimiento y sus consecuencias, realizando un diagnóstico de la situación en sus respectivos contextos, así como implementando políticas públicas tanto para la mejora de la calidad de vida de una población mayor cada vez más numerosa y diferente a la de generaciones anteriores, como para hacer frente a los retos que el aumento del peso de la población mayor significa para el conjunto de la sociedad. Entre éstos, los englobados dentro del denominado envejecimiento activo han cobrado especial relevancia durante los últimos años en el contexto europeo.

En este sentido, la Diputación Foral de Bizkaia, por iniciativa de la Dirección General de Promoción de la Autonomía Personal, aprobó en 2013 el Plan Estratégico de Personas Mayores de Bizkaia, como sucesor del Plan de Acción para la Promoción del Envejecimiento Activo en Bizkaia 2010-2011 y del Plan Gerontológico de Bizkaia 2006-2011 (Diputación Foral de Bizkaia, 2006, 2010 y 2013). Asimismo, mediante su aprobación en diciembre de 2013, el Consejo de Personas Mayores de Bizkaia se sumó a la estrategia global del territorio. Dicho plan estratégico, en su tarea de promover un envejecimiento activo de la población mayor de Bizkaia, incluye el uso del Índice de Envejecimiento Activo (Active Ageing Index) como un primer paso para mejorar el conocimiento de la situación en Bizkaia, planificar líneas de acción

Tabla 1. Población, por edad y sexo. Bizkaia, 2014

\begin{tabular}{|l|c|c|c|c|c|c|}
\hline \multirow{2}{*}{ Edad } & \multicolumn{2}{|c|}{ Total } & \multicolumn{2}{c|}{ Hombres } & \multicolumn{2}{c|}{ Mujeres } \\
\cline { 2 - 7 } & $n$ & $\%$ & $n$ & \multicolumn{2}{c|}{$\boldsymbol{n}$} & 220.789 \\
\hline$\geq 55$ años & 393.945 & 34,4 & 173.156 & 31,4 & 14,3 \\
\hline$\geq 65$ años & 242.905 & 21,2 & 100.487 & 18,2 & 24,0 \\
\hline$\geq 80$ años & 19.775 & 1,7 & 5.149 & 0,9 & 14.626 & 2,5 \\
\hline Total & 1.144 .214 & 100,0 & 551.855 & 100,0 & 592.359 & 100,0 \\
\hline
\end{tabular}

Fuente: Elaboración propia a partir de Estadística Municipal de Habitantes (Eustat, 2014a). 
coherentes con las potencialidades y carencias identificadas en torno a éste, y monitorizar los efectos de las políticas puestas en marcha. En consecuencia, quedan alineadas las estrategias del territorio histórico de Bizkaia y la Unión Europea.

Asimismo, el Gobierno Vasco, a través del Departamento de Empleo y Políticas Sociales, ha puesto en marcha la Estrategia Vasca de Envejecimiento Activo 2015-2020 (Gobierno Vasco, 2015), que tiene por finalidad ayudar a las instituciones vascas a abordar el reto demográfico desde la responsabilidad y bajo una perspectiva positiva, estableciendo para ello tres áreas temáticas:

- Adaptación de la sociedad al envejecimiento, un nuevo modelo de gobernanza.

- Anticipación y prevención para envejecer mejor.

- Amigabilidad y participación en la construcción de una sociedad del bienestar.

Ambas estrategias incluyen el uso del Índice de Envejecimiento Activo, impulsado por la Comisión Europea (2013), y cuyo marco conceptual y operativo será explicado en detalle más adelante.

\subsection{El envejecimiento activo: definición conceptual y principios sustentadores}

A finales de la década de 1990, la Organización Mundial de la Salud (OMS) comenzó a utilizar el término 'envejecimiento activo' (active ageing) en sustitución del 'envejecimiento saludable' (healthy ageing) utilizado hasta ese momento, de manera que se constituyera un concepto más inclusivo que no sólo atendiera a la salud de las personas mayores, sino que tuviese en cuenta otros factores que afectan a cómo los individuos y las poblaciones envejecen (Kalache y Kickbusch, 1997). El modelo del envejecimiento activo se basó en el reconocimiento de las personas mayores no como agentes pasivos de derechos (needs-based approach), sino como agentes activos con derecho a tener las mismas oportunidades de envejecer (rights-based approach) [OMS, 2002].

A través de su Programa de Envejecimiento y Ciclo de Vida, dicha entidad definió en 2002 el envejecimiento activo como "el proceso de optimización de las oportunidades de salud, participación y seguridad con el fin de mejorar la calidad de vida al envejecer" (OMS, 2002: 12). Esta definición implica tres dimensiones de acción política en términos de envejecimiento. En primer lugar, la salud, que se entiende de manera tridimensional, es decir, contemplando tanto la salud física, como mental y el bienestar social. En segundo lugar, la participación, que es entendida como una diversidad de actividades realizadas por las personas mayores en los ámbitos social, económico, cultural, espiritual y cívico, e incluye la participación en el mercado laboral. En tercer lugar, la OMS tiene en cuenta la seguridad de las personas mayores, que desde esta perspectiva es considerada como el acceso a un entorno seguro y saludable, tanto físico como social, a una seguridad económica y, en su caso, a un empleo gratificante.

Desde hace décadas, el incremento en la esperanza de vida que se viene observando en los países desarrollados ha enfatizado la necesidad de asegurar una mejor utilización del potencial de las personas mayores como agentes activos de la sociedad. Así, el impulso del concepto de envejecimiento activo se sustenta en la premisa del aprovechamiento de este incremento de los años de vida de la población como un recurso para el progreso social. En este sentido, 2012 fue declarado como el Año Europeo para el Envejecimiento Activo y la Solidaridad entre Generaciones (EY2012). El principal objetivo era crear conciencia del valor del envejecimiento activo, subrayando la utilidad de las contribuciones que la población mayor hace a la sociedad y a la economía, con el fin de identificar y difundir buenas prácticas, y para alentar a los/as responsables políticos y a todas las partes interesadas a promover el envejecimiento activo. Una vez finalizado el Año Europeo, el Consejo de la Unión Europea diseñó una serie de “orientaciones que han de guiar el envejecimiento activo y la solidaridad entre las generaciones" (Consejo de la Unión Europea, 2012), basadas principalmente en tres dimensiones: empleo, participación social y vida independiente.

La orientación europea del envejecimiento activo enlaza también con el marco de la Estrategia Europa 2020 para un crecimiento inteligente, sostenible e inclusivo, la cual destaca la importancia de enfrentar el reto de promover una población mayor activa y saludable que permita mayor cohesión social y mayor productividad (Comisión Europea, 2010: 18). El discurso político del envejecimiento activo, basado en utilizar el potencial de las personas mayores, está también alineado con el modelo de inversión social, que gira en torno a la idea de que la activación de ciertas políticas sociales puede reportar grandes beneficios económicos y sociales.

Dentro de este marco europeo, en 2012 se celebró en Viena la Conferencia Ministerial sobre Envejecimiento, en la que se establecieron cuatro objetivos prioritarios, que van en la línea de la definición de envejecimiento activo lanzada previamente por la OMS, y que guiarán la posterior definición conceptual y operativa del proyecto para la creación del Índice de Envejecimiento Activo como instrumento para su monitorización (Unece, 2012). Estas prioridades son:

- Fomentar la prolongación de la vida laboral y el mantenimiento de la capacidad de trabajo.

- Promover la participación, la no discriminación y la inclusión social de las personas mayores.

- Promover y salvaguardar la dignidad, la salud y la independencia entre las personas mayores.

- Mantener y mejorar la solidaridad entre generaciones. 
Durante el Año Europeo, y basándose en estos objetivos, se fue desarrollando el Índice de Envejecimiento Activo, orientado a convertirse en una de las principales herramientas para aplicar esta orientación de la inversión en políticas sociales de envejecimiento (Comisión Europea, 2013).

\subsection{El Îndice de Envejecimiento Activo (Active Ageing Index)}

La Unión Europea incluyó el diagnóstico de la situación de la población mayor en los 28 países miembros dentro de la agenda europea para el envejecimiento activo, lanzándose así el proyecto Active Ageing Index (Índice de Envejecimiento Activo), gestionado conjuntamente por la Comisión Europea, a través de la Dirección General de Empleo, Asuntos Sociales e Inclusión, y la Unidad de Población de la Comisión Económica para Europa de Naciones Unidas (Unece). La definición de envejecimiento activo adoptada por el proyecto fue discutida en dos encuentros del Grupo de Expertos/as que tuvieron lugar durante 2012, y que, siguiendo las prioridades marcadas por la Comisión y los elementos claves destacados por la OMS, concluyeron que:

El envejecimiento activo hace referencia a la situación en la que las personas continúan participando en el mercado laboral formal, así como también se comprometen en otras actividades productivas no remuneradas (tales como la provisión de cuidados a los miembros de la familia y el voluntariado) y viven de forma saludable, independiente y segura a medida que envejecen (Zaidi et al., 2013: 6)' .

A partir de esta definición, se construyó un índice compuesto de cuatro dimensiones, tres de las cuales respondían a la voluntad de recoger la experiencia de envejecimiento activo de la población mayor (empleo, participación social y vida independiente), y una cuarta que pretendía captar la capacidad tanto de las personas como del entorno en el que viven para promover un envejecimiento saludable y activo. Esta dimensión se basó en el modelo de Amartya Sen (1993, 2009) sobre las capacidades (capabilities), definidas como oportunidades sustantivas y empoderadoras para mejorar el bienestar y la calidad de vida de las personas. Así, la cuarta dimensión del índice trarta de reflejar los activos humanos (esperanza de vida), el capital de salud (esperanza de vida en buena salud física y mental) y el capital humano (nivel educativo) de las personas de cara a favorecer procesos de envejecimiento activos.

El Índice de Envejecimiento Activo se ha establecido como una herramienta de medición comparada entre países del potencial de las personas mayores para tener un envejecimiento activo y saludable. El índice mide el nivel de vida independiente que tienen las personas mayores, su participación en el trabajo

${ }^{1}$ Traducción propia. remunerado y en actividades sociales, así como su capacidad para envejecer activamente. El índice se compone de 22 indicadores que se agrupan en cuatro dimensiones: empleo, participación social, vida independiente y segura, y capacidad para un envejecimiento saludable. Desde 2010 y cada dos años, se ha calculado para cada país de la Unión Europea (UE-28), proporcionando así una panorámica de la situación de envejecimiento activo en cada país y de su evolución. Además, algunas regiones europeas se han sumado al proyecto realizando el cálculo del índice a escala subestatal. El objetivo de este artículo es presentar los resultados del Índice de Envejecimiento Activo para el territorio histórico de Bizkaia, ofreciendo un diagnóstico de la situación de su población mayor (de 55 y más años), e identificando posibles carencias y desigualdades en el desarrollo de un envejecimiento activo.

\section{Metodología del estudio}

El cálculo del índice global y de las puntuaciones para cada dimensión en Bizkaia ha seguido la metodología del índice diseñada a escala europea (Zaidi et al., 2013), si bien se aplicaron dos estrategias consecutivas. En primer lugar, se utilizó información de bases de datos secundarias para calcular los indicadores del índice, analizando su comparabilidad con los indicadores originales. Posteriormente, se complementó el cálculo de los indicadores que tenían mayores problemas de comparabilidad mediante la producción de datos primarios a través de una encuesta a población de 55 y más años residente en Bizkaia, realizada ad hoc y que se ha denominado Encuesta de Envejecimiento Activo en Bizkaia 2014. De esta manera, combinando ambas estrategias y en función de la mejor adaptación de cada fuente de datos al cálculo de cada indicador (comparabilidad y literalidad de las preguntas, año de la fuente de datos, tamaño muestral), el Índice de Envejecimiento Activo se ha calculado utilizando datos primarios para 12 de los 22 indicadores de los que se compone y datos secundarios para los 10 indicadores restantes. En el anexo, se detallan las fuentes de datos, años y tamaño de la muestra para la población de Bizkaia en relación con cada uno de los 22 indicadores.

En cuanto a la fuente de datos primaria, la Encuesta de Envejecimiento Activo en Bizkaia 2014, se elaboró un cuestionario siguiendo las preguntas originales que se recogen en la metodología europea (Zaidi et al., 2013). Para la selección de los individuos, se contó con un fichero de 15.000 hogares de Bizkaia, creado por DataCentric PDM S.A. a partir de fuentes accesibles al público, en particular de las guías de abonados/as a servicios de telecomunicaciones. Así, se eligió una muestra por cuotas de edad y sexo, teniendo en cuenta la distribución de la población de Bizkaia de 55 y más años. Se realizaron un total de 1.362 entrevistas telefónicas mediante el sistema Computer Assisted Telephone Interview (CATI), que, suponiendo un muestreo aleatorio simple y la máxima heterogeneidad $(p=q)$, conllevaba, un margen de error de $\pm 2,65 \%$ y un nivel de confianza 
de $95 \%$. La encuesta se realizó entre el 6 y el 18 de noviembre de 2014 en el centro de llamadas de Zugaztel. El trabajo de campo fue coordinado por la empresa Gaia-Investigación y Consultoría.

Las fuentes datos secundarias sobre la población mayor de Bizkaia fueron facilitadas por el EustatInstituto Vasco de Estadística. En concreto, se utilizaron la Encuesta de Salud del País Vasco 2013, la Encuesta de Población en Relación con la Actividad del País Vasco 2012-2013, la Encuesta Demográfica del País Vasco 2011, la Encuesta de Pobreza y Desigualdades Sociales del País Vasco 2012, la Encuesta sobre la Sociedad de la Información del País Vasco 2013, la Encuesta sobre Capital Social del País Vasco 2012 y la Estadística de Mortalidad del País Vasco.

Una vez seleccionadas las fuentes de datos y calculados los indicadores, se realizó el cálculo de las cuatro dimensiones, según la ponderación de cada indicador dentro de la dimensión, y del índice global, que es el resultado de la suma ponderada de las cuatro dimensiones: 0,35 para la primera y segunda dimensión, 0,10 para la tercera y 0,20 para la cuarta. Esta ponderación es coherente con la priorización del empleo remunerado y de las actividades no remuneradas y de voluntariado como elementos centrales para un envejecimiento activo, según la definición adoptada por el proyecto del índice. La Tabla 2 muestra la batería de indicadores para cada dimensión, así como los pesos asignados para ponderar los indicadores dentro de las dimensiones y de cada dimensión dentro el índice global. Todos los cálculos se realizaron de forma separada para hombres y mujeres.

\section{Resultados}

A continuación se describen los resultados para Bizkaia del Índice de Envejecimiento Activo, de cada dimensión y de los indicadores que lo componen, de forma comparada con el resto de países de la UE-28, destacando la posición de Bizkaia respecto a la media europea y al país con mejores puntuaciones.

Tabla 2. Índice de Envejecimiento Activo: dimensiones, indicadores y pesos asignados

\begin{tabular}{|c|c|c|}
\hline Dimensión & Indicador & Pesos \\
\hline \multirow{5}{*}{ 1. Empleo } & 1.1. Tasa de ocupación en población de 55 a 59 años & 25,0 \\
\hline & 1.2. Tasa de ocupación en población de 60 a 64 años & 25,0 \\
\hline & 1.3. Tasa de ocupación en población de 65 a 69 años & 25,0 \\
\hline & 1.4. Tasa de ocupación en población de 70 a 74 años & 25,0 \\
\hline & Total & 35,0 \\
\hline \multirow{5}{*}{$\begin{array}{l}\text { 2. Participación } \\
\text { social }\end{array}$} & 2.1. Porcentaje de población $\geq 55$ años que realiza trabajo voluntario en asociaciones & 25,0 \\
\hline & 2.2. Porcentaje de población $\geq 55$ años que cuida a hijos/as o nietos/as al menos 1 vez/semana & 25,0 \\
\hline & $\begin{array}{l}\text { 2.3. Porcentaje de población } \geq 55 \text { años que cuida a personas mayores o discapacitadas al menos } 1 \text { vez/ } \\
\text { semana }\end{array}$ & 30,0 \\
\hline & $\begin{array}{l}\text { 2.4. Porcentaje de población } \geq 55 \text { años que participa en las actividades de un sindicato, partido político u } \\
\text { otro grupo con actividad política }\end{array}$ & 20,0 \\
\hline & Total & 35,0 \\
\hline \multirow{9}{*}{$\begin{array}{l}\text { 3. Vida } \\
\text { independiente y } \\
\text { segura }\end{array}$} & 3.1. Porcentaje de población $\geq 55$ años que realiza actividad física o deporte al menos 5 veces/semana & 10,0 \\
\hline & $\begin{array}{l}\text { 3.2. Porcentaje de población } \geq 55 \text { años que afirma no haber tenido necesidades médicas o dentales no } \\
\text { cubiertas (examen o tratamiento) en los últimos } 12 \text { meses }\end{array}$ & 20,0 \\
\hline & 3.3. Porcentaje de población $\geq 75$ años que vive en una vivienda unipersonal o en pareja & 20,0 \\
\hline & $\begin{array}{l}\text { 3.4. Ratio entre la renta mediana disponible equivalente de la población } \geq 65 \text { años y la de la población }<65 \\
\text { años }\end{array}$ & 10,0 \\
\hline & 3.5. Porcentaje de población $\geq 65$ años que no se encuentra en riesgo de pobreza & 10,0 \\
\hline & 3.6. Porcentaje de población $\geq 65$ años sin privación material severa & 10,0 \\
\hline & 3.7. Porcentaje de población $\geq 55$ años que no está preocupada por ser víctima de un delito violento & 10,0 \\
\hline & $\begin{array}{l}\text { 3.8. Porcentaje de población de } 55 \text { a } 74 \text { años que afirma haber recibido algún tipo de formación el mes } \\
\text { anterior }\end{array}$ & 10,0 \\
\hline & Total & 10,0 \\
\hline \multirow{7}{*}{$\begin{array}{l}\text { 4. Capacidad } \\
\text { para un } \\
\text { envejecimiento } \\
\text { activo }\end{array}$} & $\begin{array}{l}\text { 4.1. Esperanza de vida a los } 55 \text { años dividida entre 50, para calcular la proporción de esperanza de vida } \\
\text { alcanzada sobre el objetivo de } 105 \text { años }\end{array}$ & 33,3 \\
\hline & 4.2. Porcentaje de esperanza de salud sobre la esperanza de vida total a los 55 años & 23,3 \\
\hline & 4.3. Salud mental en población $\geq 55$ años & 16,7 \\
\hline & 4.4. Porcentaje de población de 55-74 años que utiliza internet al menos 1 vez/semana & 6,7 \\
\hline & 4.5. Porcentaje de población $\geq 55$ años con contacto diario o frecuente con amistades, familiares o colegas & 13,3 \\
\hline & 4.6. Porcentaje de población de 55 a 74 años con educación secundaria superior o terciaria & 6,7 \\
\hline & Total & 20,0 \\
\hline
\end{tabular}




\subsection{Posición comparada de Bizkaia dentro de la Unión Europea en el índice global y en cada dimensión}

Bizkaia obtiene una puntuación en el Îndice de Envejecimiento Activo de 37,9 puntos en hombres y de 34,7 en mujeres, situándose por encima de la media de la Unión Europea (UE-28) en ambos casos. Como se aprecia en el Gráfico 1, la distancia de Bizkaia con la media de la Unión Europea es destacable, y en mayor medida, también con el Estado. Así, la puntuación del índice global en los hombres de Bizkaia es 1,9 puntos superior a la media de la UE-28 y 3,6 puntos superior a la del conjunto del Estado español, mientras que en las mujeres la distancia es mayor, de 2,5 puntos con la UE-28 y de 3,3 con el Estado.

A pesar de esta buena posición comparativa con el conjunto de la Unión, también es reseñable la distancia que existe entre la puntuación de Bizkaia y los países que se encuentran en una mejor situación de envejecimiento activo. Así, existen casi 9 puntos de diferencia, tanto en hombres como en mujeres, con el país de mayor puntuación, que en ambos sexos es Suecia.

Si observamos las diferencias por sexo, comparativamente con el resto de países, las mujeres de Bizkaia puntúan, en términos relativos, mejor que los hombres, colocándose en la octava posición, igualadas con las mujeres francesas. Sin embargo, atendiendo a las diferencias entre sexos, dentro de Bizkaia las mujeres obtienen 3,2 puntos menos que los hombres, lo cual muestra que su situación de envejecimiento activo es moderadamente peor que la de sus pares masculinos. Como se aprecia en el Gráfico 2, esta diferencia en el índice se repite en la mayoría de los países de la UE-28, exceptuando Estonia, Letonia y Finlandia, donde las mujeres tienen una puntuación ligeramente más alta que los hombres.

Gráfico 1. Resultados globales del Îndice de Envejecimiento Activo. Países de la UE-28, media de la UE-28 y Bizkaia

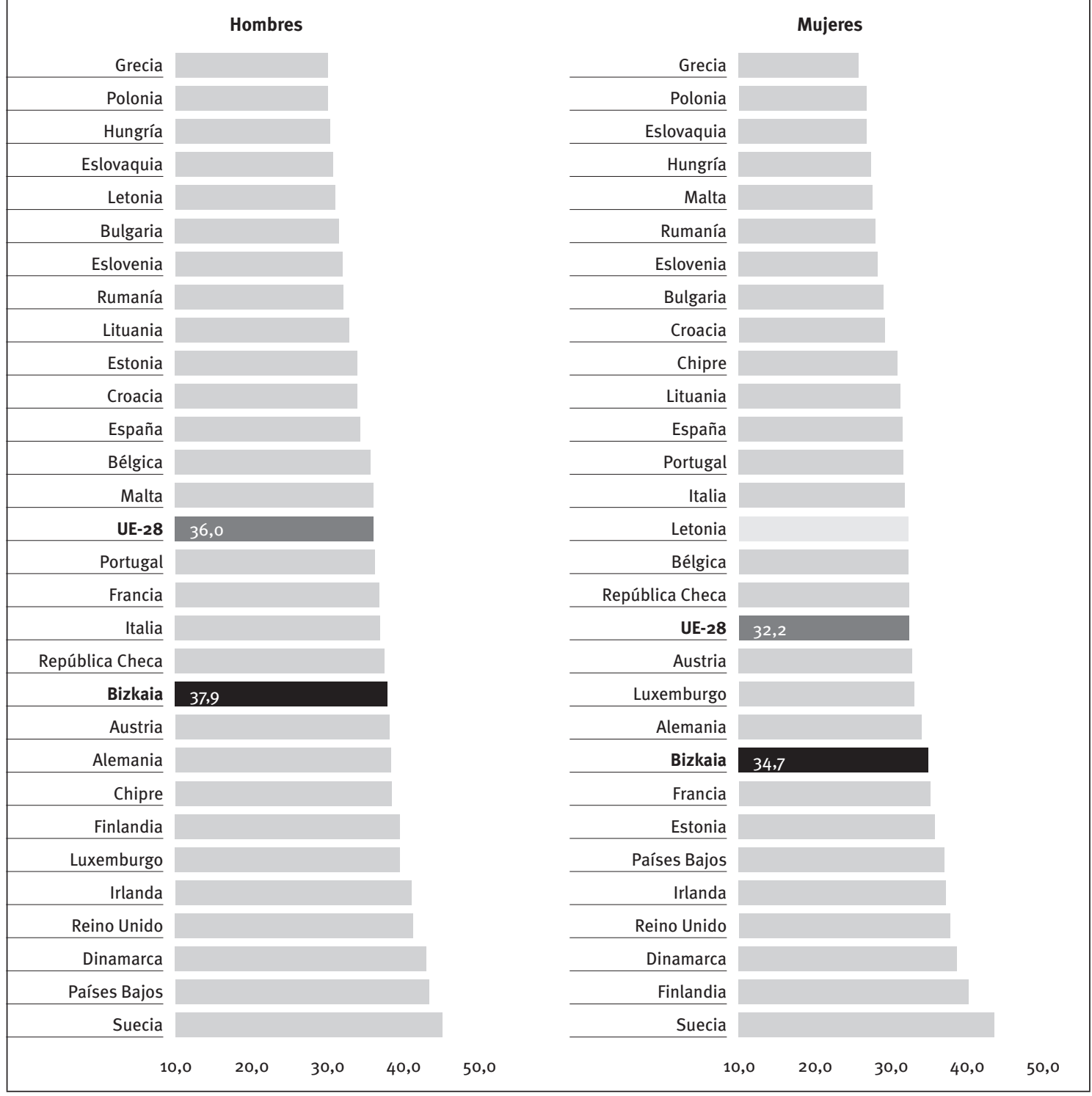

Fuente: Elaboración propia. 


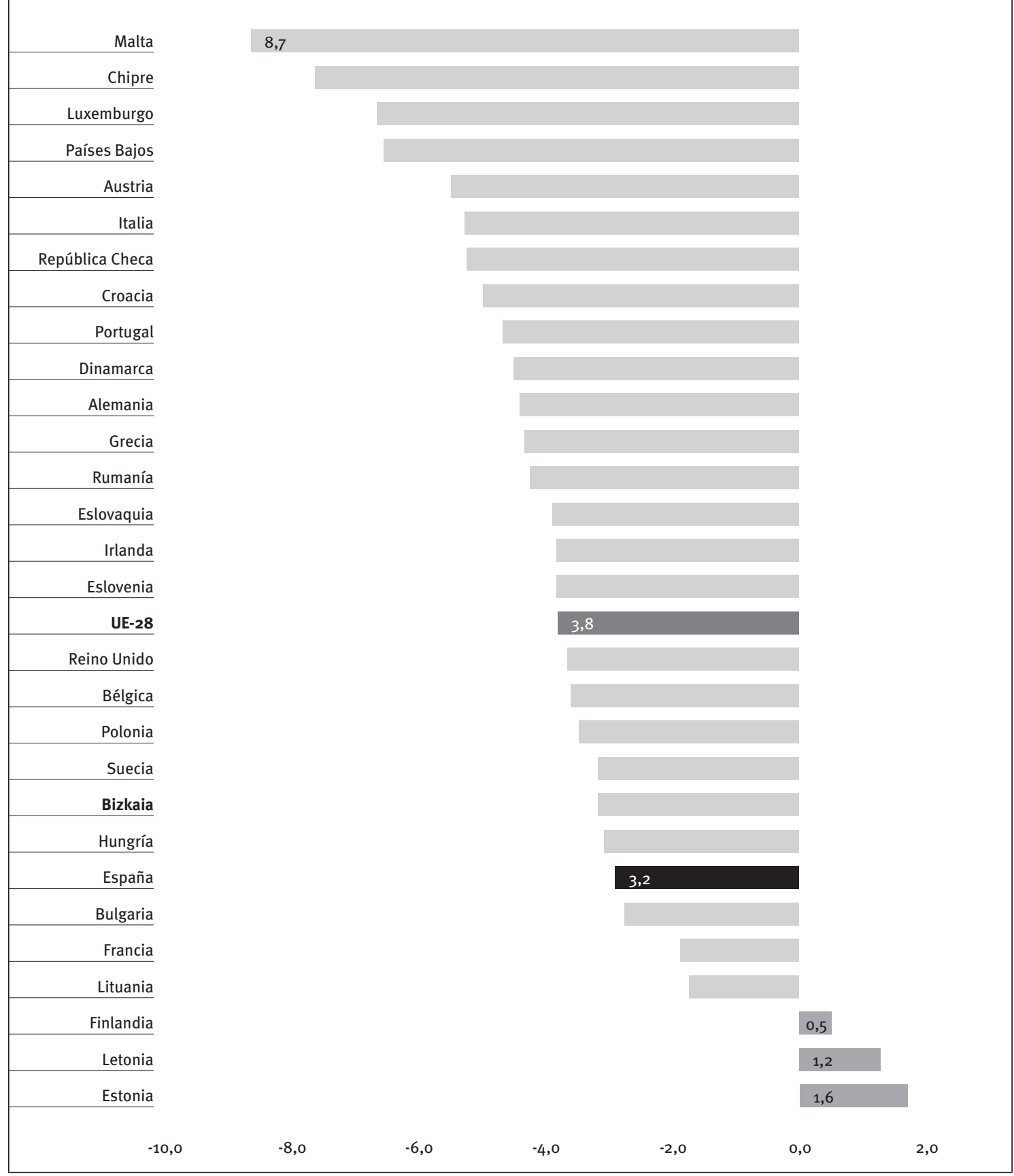

Fuente: Elaboración propia.

Al analizar por separado las cuatro dimensiones, observamos que la posición de Bizkaia en cada una de ellas es diferente (Gráfico 3). En la dimensión relativa al empleo, Bizkaia obtiene un valor más bajo que la media de la UE-28, tanto en hombres como en mujeres. Además, es notoria la diferencia en la tasa de ocupación de hombres y mujeres en Bizkaia, 10 puntos más baja en las mujeres (19,2\%). También en la dimensión relativa a vida independiente y segura, Bizkaia muestra valores ligeramente inferiores a la media de la UE-28 en los hombres (6 puntos menos), aunque en el caso de las mujeres su puntuación $(68,9)$ es muy similar a la media euro- pea $(69,4)$. Por otro lado, en la dimensión relativa a la participación social de la población mayor de 54 años, Bizkaia alcanza una puntuación alta en los cuatro indicadores y, por tanto, en el conjunto de la dimensión. De hecho, la participación social de la población de Bizkaia es moderadamente más alta que la media europea tanto en hombres $(23,2)$ como en mujeres $(24,1)$. Finalmente, en la dimensión sobre la capacidad de envejecer activamente, la posición de Bizkaia es aventajada, situándose casi 10 puntos por encima de la media europea tanto en hombres como en mujeres, y a escasa distancia de Suecia, el país de mayor puntuación. 


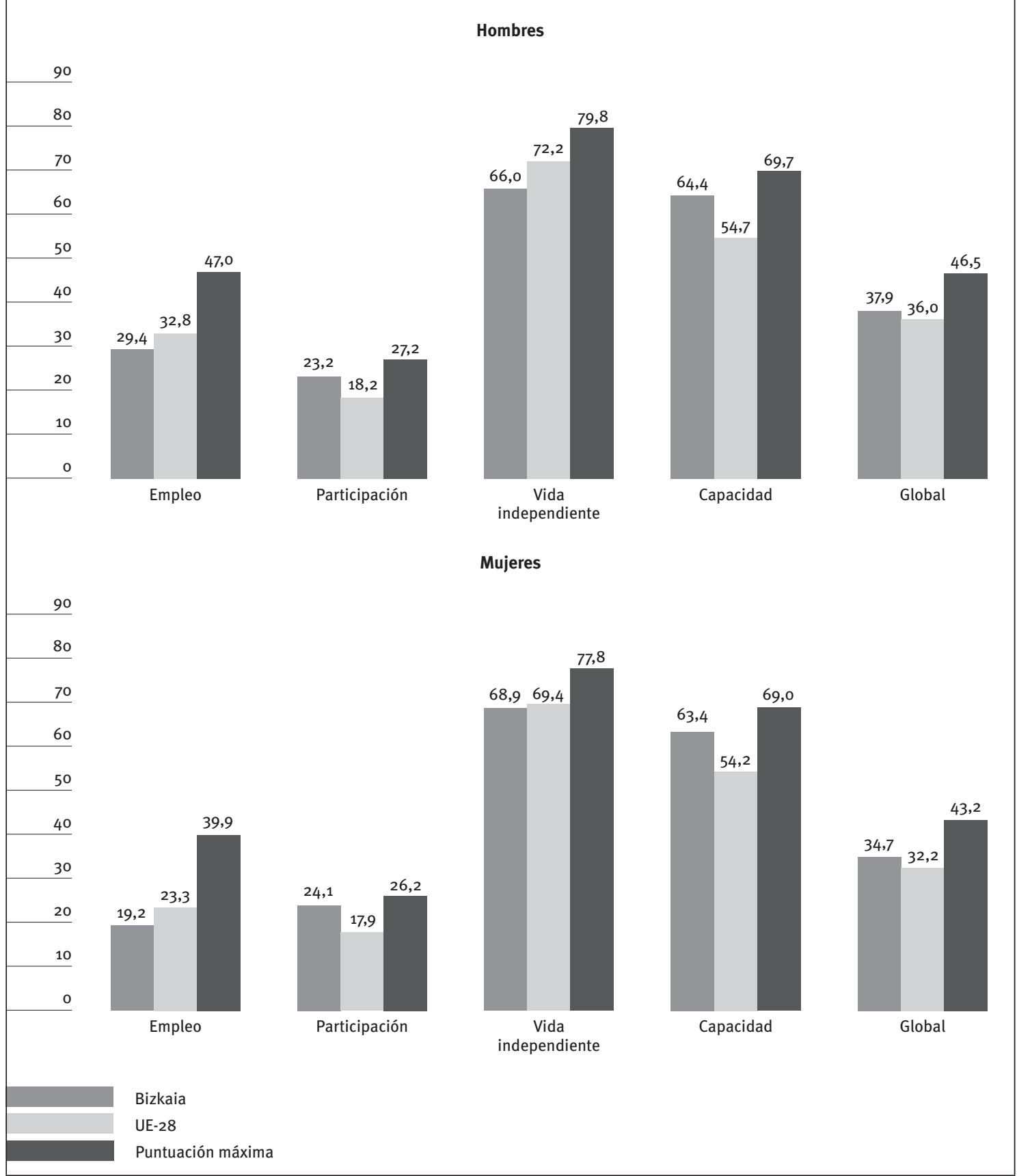

Fuente: Elaboración propia.

\subsection{Explicando la puntuación de Bizkaia en cada dimensión: indicadores más destacados}

Como se ha mostrado, Bizkaia registra altas puntuaciones en dos de las dimensiones del índice, mientras que en otras dos su situación es comparativamente peor que la media de la Unión Europea.

Empezando por aquellos indicadores peor posicionados, se van a analizar en primer lugar las tasas de ocupación (Tabla 3). La ocupación de los mayores de 64 años en Bizkaia es sensiblemente más baja que la media europea, tanto en hombres como en mujeres. Además, las tasas de ocupación de los cuatro grupos de edad son mucho menores en mujeres que en hombres, al igual que ocurre en la mayoría de los países europeos. En Bizkaia, la ocupación de los hombres en los grupos de edad de 55 a 59 y de 60 a 64 años son similares e incluso ligeramente superiores a la media de la UE-28, pero entre los de más de 65 años desciende a niveles muy bajos. Ello explica la baja puntuación de la dimensión en su conjunto, que se encuentra a tres puntos de la media europea y muy lejos de Suecia, el país con la máxima puntuación entre los hombres. 
Entre las mujeres, la ocupación entre los 55 y los 59 años es menor que la media de la UE-28, así como también en el grupo de 65 y más años, mientras que es ligeramente más alta en el grupo de 60 a 64 años. De esta manera, la puntuación de la dimensión en Bizkaia es, también en las mujeres, 4 puntos más baja que la media europea y asimismo alejada de Estonia, el país con la tasa de ocupación femenina más alta.

Por otro lado, Bizkaia destaca por su peor posición relativa en algunos indicadores de la tercera dimensión, 'vida independiente y segura' (Tabla 4). En los hombres, ello se debe fundamentalmente al bajo porcentaje de mayores de 74 años que viven autónomamente, es decir, solos o en pareja, un 39,2\%, en comparación con la media de la UE-28, que asciende al 84,2\%. Entre las mujeres, este indicador es también más bajo que la media europea, pero a menos distancia que en el caso de los hombres
$(62,7 \%$ en Bizkaia y $84,2 \%$ en Europa). Otro indicador que sobresale negativamente es el referido a la población que afirma no haber tenido necesidades médicas o dentales no cubiertas en los últimos 12 meses, siendo menor en Bizkaia $(77,1 \%$ en hombres y $76,5 \%$ en mujeres) que en la UE-28 (88,6\% y $87,8 \%$ respectivamente). Por último, es destacable el menor dato para Bizkaia de la ratio de renta disponible para la población mayor de 65 respecto a la población menor $(84,4 \%$ en hombres y $72,9 \%$ en mujeres), frente a la media europea ( $91,2 \%$ en hombres y $84 \%$ en mujeres). Así, a pesar de que Bizkaia puntúa de manera similar a la media europea en el resto de indicadores de esta dimensión, o incluso por encima en algunos de ellos, el mayor peso otorgado dentro de la dimensión a los dos primeros indicadores mencionados (20 puntos) respecto al resto (10 puntos) supone una menor puntuación de Bizkaia en el conjunto de la dimensión.

Tabla 3. Resultados del Índice de Envejecimiento Activo en la dimensión 'empleo’. Bizkaia, UE-28 y país de la UE-28 con la máxima puntuación

\begin{tabular}{|l|c|c|c|c|c|c|}
\hline \multirow{2}{*}{ Indicadores } & \multicolumn{3}{|c|}{ Hombres } & \multicolumn{2}{c|}{ Mujeres } \\
\cline { 2 - 7 } & Bizkaia & UE-28 & Máxima & Bizkaia & UE-28 & Máxima \\
\hline $\begin{array}{l}\text { Tasa de ocupación en población de 55 } \\
\text { a 59 años }\end{array}$ & 72,0 & 69,3 & $\begin{array}{c}84,3 \\
\text { (Suecia) }\end{array}$ & 47,1 & 55,3 & $\begin{array}{c}79,6 \\
\text { (Suecia) }\end{array}$ \\
\hline $\begin{array}{l}\text { Tasa de ocupación en población de 60 } \\
\text { a 64 años }\end{array}$ & 40,0 & 38,6 & $\begin{array}{c}68,4 \\
\text { (Suecia) }\end{array}$ & 26,8 & 25,1 & $\begin{array}{c}59,9 \\
\text { (Suecia) }\end{array}$ \\
\hline $\begin{array}{l}\text { Tasa de ocupación en población de 64 } \\
\text { a 69 años }\end{array}$ & 3,4 & 15,1 & $\begin{array}{c}28,5 \\
\text { (Portugal) }\end{array}$ & 2,3 & 8,5 & $\begin{array}{c}26,7 \\
\text { (Estonia) }\end{array}$ \\
\hline $\begin{array}{l}\text { Tasa de ocupación en población de 70 } \\
\text { a 74 años }\end{array}$ & 2,3 & 8,2 & $\begin{array}{c}24,1 \\
\text { (Portugal) }\end{array}$ & 0,6 & 4,3 & $\begin{array}{c}\text { 19,6 } \\
\text { (Rumanía) }\end{array}$ \\
\hline Total & $\mathbf{2 9 , 4}$ & $\mathbf{3 2 , 8}$ & $\begin{array}{c}\mathbf{4 7 , 0} \\
\text { (Suecia) }\end{array}$ & $\mathbf{1 9 , 2}$ & $\mathbf{2 3 , 3}$ & $\begin{array}{c}\mathbf{4 0 , 2} \\
\text { (Estonia) }\end{array}$ \\
\hline
\end{tabular}

Fuente: Elaboración propia.

Tabla 4. Resultados del Índice de Envejecimiento Activo en la dimensión 'vida independiente y segura'. Bizkaia, UE-28 y país de la UE-28 con la máxima puntuación

\begin{tabular}{|c|c|c|c|c|c|c|}
\hline \multirow{2}{*}{ Indicadores } & \multicolumn{3}{|c|}{ Hombres } & \multicolumn{3}{|c|}{ Mujeres } \\
\hline & Bizkaia & UE-28 & Máxima & Bizkaia & UE-28 & Máxima \\
\hline $\begin{array}{l}\text { Porcentaje de población } \geq 55 \text { años que realiza actividad física o } \\
\text { deporte al menos } 5 \text { veces/semana }\end{array}$ & 58,8 & 16,0 & 47,0 (Finlandia) & 50,8 & 15,2 & $\begin{array}{l}50,4 \\
\text { (Finlandia) }\end{array}$ \\
\hline $\begin{array}{l}\text { Porcentaje de población } \geq 55 \text { años que afirma no haber tenido } \\
\text { necesidades médicas o dentales no cubiertas (examen o } \\
\text { tratamiento) en los últimos } 12 \text { meses }\end{array}$ & 77,1 & 88,6 & $\begin{array}{c}98,3 \\
\text { (Países Bajos) }\end{array}$ & 76,5 & 87,8 & $\begin{array}{l}97,6 \\
\text { (Eslovenia) }\end{array}$ \\
\hline $\begin{array}{l}\text { Porcentaje de población } \geq 75 \text { años que vive en una vivienda } \\
\text { unipersonal o en pareja }\end{array}$ & 39,2 & 84,2 & $\begin{array}{c}99,6 \\
\text { (Dinamarca) }\end{array}$ & 62,7 & 84,2 & $\begin{array}{c}99,4 \\
\text { (Dinamarca) }\end{array}$ \\
\hline $\begin{array}{l}\text { Ratio entre la renta mediana disponible equivalente de la } \\
\text { población } \geq 65 \text { años y la de la población }<65 \text { años }\end{array}$ & 84,4 & 91,2 & $\begin{array}{c}115,1 \\
\text { (Luxemburgo) }\end{array}$ & 72,9 & 84,0 & $\begin{array}{c}107,1 \\
\text { (Luxemburgo) }\end{array}$ \\
\hline $\begin{array}{l}\text { Porcentaje de población } \geq 65 \text { años que no se encuentra en riesgo } \\
\text { de pobreza }\end{array}$ & 92,0 & 94,6 & $\begin{array}{l}99,3 \\
\text { (República } \\
\text { Checa) }\end{array}$ & 90,0 & 92,0 & $\begin{array}{l}98,2 \\
\text { (República } \\
\text { Checa) }\end{array}$ \\
\hline Porcentaje de población $\geq 65$ años sin privación material severa & 96,0 & 91,7 & $\begin{array}{c}100,0 \\
\text { (Luxemburgo) }\end{array}$ & 92,7 & 88,9 & $\begin{array}{c}100,0 \\
\text { (Luxemburgo) }\end{array}$ \\
\hline $\begin{array}{l}\text { Porcentaje de población } \geq 55 \text { años que no está preocupada por } \\
\text { ser víctima de un delito violento }\end{array}$ & 83,0 & 78,0 & $\begin{array}{c}98,1 \\
\text { (Eslovenia) }\end{array}$ & 84,3 & 61,8 & $\begin{array}{c}92,2 \\
\text { (Eslovenia) }\end{array}$ \\
\hline $\begin{array}{l}\text { Porcentaje de población } 55 \text { a } 74 \text { años que afirma haber recibido } \\
\text { algún tipo de formación el mes anterior }\end{array}$ & 13,5 & 3,4 & $\begin{array}{c}15,4 \\
\text { (Dinamarca) }\end{array}$ & 19,8 & 5,5 & $\begin{array}{c}30,1 \\
\text { (Dinamarca) }\end{array}$ \\
\hline Total & 66,0 & 72,2 & $\begin{array}{c}\mathbf{8 0 , 2} \\
\text { (Finlandia) }\end{array}$ & 68,9 & 69,4 & $\begin{array}{c}78,9 \\
\text { (Dinamarca) }\end{array}$ \\
\hline
\end{tabular}

Fuente: Elaboración propia. 
En cuanto a los indicadores en los que Bizkaia destaca por su posición más favorable, son remarcables aquellos relacionados con la participación social (Tabla 5). Así, el trabajo voluntario entre la población mayor de Bizkaia es más frecuente que en otros países de la UE, llegando al $19 \%$ en hombres y al $20,8 \%$ en mujeres. También la población de Bizkaia destaca frente al resto de países en los indicadores sobre cuidados, como es el caso del cuidado a familiares mayores o con discapacidad, que en Bizkaia obtiene la mayor puntuación de toda la UE-28. Sin embargo, en este indicador se aprecia una importante brecha entre ambos sexos, con un valor más alto en el caso de las mujeres (24\%) que entre los hombres (18,2\%). En relación al cuidado a hijos/as o nietos/as, Bizkaia también que se sitúa muy por encima de la media europea, tanto en hombres como en mujeres. Por último, en lo referido a la participación política y sindical, Bizkaia también queda por encima de la media europea, siendo, sin embargo, mucho menor en mujeres (11\%) que en hombres (19,2\%).
Finalmente, es destacable también la posición de Bizkaia en términos de la capacidad de envejecimiento activo de su población mayor, esto es, en aquellos indicadores relacionados con la salud y las actividades cotidianas (Tabla 6). Así, los hombres y mujeres de Bizkaia tienen la mayor esperanza de vida a los 55 años, y una alta proporción de años en buena salud, por encima de la media de la Unión Europea, tanto en hombres (68,7\%) como en mujeres (57,5\%). Además, la salud mental de los hombres y mujeres de Bizkaia es muy buena, estando de hecho más de veinte puntos por encima de la media europea y a la cabeza en el caso de las mujeres $(86,5 \%)$. Por otro lado, en términos de la relación con el entorno de la población mayor, se aprecia, en primer lugar que el uso de nuevas tecnologías en Bizkaia es también superior al valor medio de la UE-28, y sin embargo, queda muy lejos de los países en los que su población utiliza internet en mayor medida, principalmente los nórdicos, el Reino Unido y Alemania; y en segundo lugar, que el contacto frecuente con amista-

Tabla 5. Resultados del Î́ndice de Envejecimiento Activo en la dimensión 'participación social'. Bizkaia, UE-28 y país de la UE-28 con la máxima puntuación

\begin{tabular}{|c|c|c|c|c|c|c|}
\hline \multirow{2}{*}{ Indicadores } & \multicolumn{3}{|c|}{ Hombres } & \multicolumn{3}{|c|}{ Mujeres } \\
\hline & Bizkaia & UE-28 & Máxima & Bizkaia & UE-28 & Máxima \\
\hline $\begin{array}{l}\text { Porcentaje de población } \geq 55 \text { años que realiza trabajo voluntario } \\
\text { en asociaciones }\end{array}$ & 19,0 & 15,8 & $\begin{array}{c}39,4 \\
\text { (Austria) }\end{array}$ & 20,8 & 13,8 & $\begin{array}{l}30,0 \\
\text { (Países Bajos) }\end{array}$ \\
\hline $\begin{array}{l}\text { Porcentaje de población } \geq 55 \text { años que cuida a hijos/as o } \\
\text { nietos/as al menos } 1 \text { vez/semana }\end{array}$ & 36,7 & 30,6 & $\begin{array}{l}49,9 \\
\text { (Italia) }\end{array}$ & 38,0 & 33,9 & $\begin{array}{l}56,8 \\
\text { (Italia) }\end{array}$ \\
\hline $\begin{array}{l}\text { Porcentaje de población } \geq 55 \text { años que cuida a personas } \\
\text { mayores o discapacitadas al menos } 1 \text { vez/semana }\end{array}$ & 18,2 & 11,8 & $\begin{array}{l}17,6 \\
\text { (República } \\
\text { Checa) }\end{array}$ & 24,0 & 13,7 & $\begin{array}{c}20,1 \\
\text { (Irlanda) }\end{array}$ \\
\hline $\begin{array}{l}\text { Porcentaje de población } \geq 55 \text { años que participa en las } \\
\text { actividades de un sindicato, partido político u otro grupo con } \\
\text { actividad política }\end{array}$ & 19,2 & 15,5 & $\begin{array}{c}38,9 \\
\text { (Luxemburgo) }\end{array}$ & 11,1 & 9,1 & $\begin{array}{c}26,9 \\
\text { (Suecia) }\end{array}$ \\
\hline Total & 23,2 & 18,2 & $\begin{array}{l}27,2 \\
\text { (Luxemburgo) }\end{array}$ & 24,1 & 17,9 & $\begin{array}{l}26,2 \\
\text { (Irlanda) }\end{array}$ \\
\hline
\end{tabular}

Fuente: Elaboración propia.

Tabla 6. Resultados del Índice de Envejecimiento Activo en la dimensión 'capacidad para un envejecimiento activo'. Bizkaia, UE-28 y país de la UE-28 con la máxima puntuación

\begin{tabular}{|c|c|c|c|c|c|c|}
\hline \multirow{2}{*}{ Indicadores } & \multicolumn{3}{|c|}{ Hombres } & \multicolumn{3}{|c|}{ Mujeres } \\
\hline & Bizkaia & UE-28 & Máxima & Bizkaia & UE-28 & Máxima \\
\hline $\begin{array}{l}\text { Esperanza de vida a los } 55 \text { años dividida entre } 50 \text {, para calcular } \\
\text { la proporción de esperanza de vida alcanzada sobre el objetivo } \\
\text { de } 105 \text { años }\end{array}$ & 54,3 & 48,8 & $\begin{array}{c}54,0 \\
\text { (Suecia) }\end{array}$ & 66,4 & 58,1 & $\begin{array}{c}64,4 \\
\text { (Francia) }\end{array}$ \\
\hline $\begin{array}{l}\text { Porcentaje de esperanza de salud sobre la esperanza de vida } \\
\text { total a los } 55 \text { años }\end{array}$ & 68,7 & 57,1 & $\begin{array}{c}78,5 \\
\text { (Suecia) }\end{array}$ & 57,5 & 50,1 & $\begin{array}{c}74,0 \\
\text { (Suecia) }\end{array}$ \\
\hline Salud mental en población $\geq 55$ años & 89,6 & 68,2 & $\begin{array}{l}90,2 \\
\text { (Dinamarca) }\end{array}$ & 86,5 & 61,6 & $\begin{array}{c}84,6 \\
\text { (Dinamarca) }\end{array}$ \\
\hline $\begin{array}{l}\text { Porcentaje de población de } 55 \text { a } 74 \text { años que utiliza internet al } \\
\text { menos } 1 \text { vez/semana }\end{array}$ & 53,9 & 44,8 & $\begin{array}{c}85,0 \\
\text { (Luxemburgo) }\end{array}$ & 39,5 & 37,1 & $\begin{array}{c}74,0 \\
\text { (Suecia) }\end{array}$ \\
\hline $\begin{array}{l}\text { Porcentaje de población } \geq 55 \text { años con contacto diario o } \\
\text { frecuente con amistades, familiares o colegas }\end{array}$ & 67,4 & 48,0 & $\begin{array}{l}76,0 \\
\text { (Portugal) }\end{array}$ & 67,9 & 50,0 & $\begin{array}{c}72,1 \\
\text { (Portugal) }\end{array}$ \\
\hline $\begin{array}{l}\text { Porcentaje de población de } 55 \text { a } 74 \text { años con educación } \\
\text { secundaria superior o terciaria }\end{array}$ & 41,3 & 64,9 & $\begin{array}{l}92,3 \\
\text { (República } \\
\text { Checa) }\end{array}$ & 25,7 & 55,1 & $\begin{array}{c}82,5 \\
\text { (Estonia) }\end{array}$ \\
\hline Total & 64,4 & 54,7 & $\begin{array}{c}69,7 \\
\text { (Suecia) }\end{array}$ & 57,5 & 50,1 & $\begin{array}{c}74 \\
\text { (Suecia) }\end{array}$ \\
\hline
\end{tabular}

Fuente: Elaboración propia. 
des o familiares es muy alto (67\%), lo cual contribuye a generar y mantener una intensa red social de apoyo. Por último, dentro de los indicadores sobre la capacidad para envejecer activamente de la población encontramos el nivel educativo, que en Bizkaia, dada la tardía universalización de la educación, conlleva proporciones de niveles educativos secundario o superiores mucho más bajos que la media de la Unión Europea, de forma que queda a gran distancia de los países más avanzados en este sentido, como es el caso de los antiguos países del Bloque del Este.

\section{Conclusiones}

El envejecimiento de la población es un reto social y político de primer orden en las sociedades occidentalizadas. Europa tiene una importante población mayor que va a continuar creciendo en el futuro. Ante esta situación, la Unión Europea está promoviendo políticas que contribuyan a mejorar no sólo la calidad de vida y el bienestar de una población mayor cada vez más numerosa, sino también a fomentar la continuidad de su participación activa en la sociedad. De este interés surge el proyecto del Índice de Envejecimiento Activo, cuyo objetivo es, por un lado, realizar un diagnóstico de la situación de la población mayor en lo relativo a su participación productiva y social, y a su capacidad para envejecer activamente; y por otro lado, monitorizar los efectos de las políticas públicas desarrolladas para afrontar el envejecimiento de la población y promover su productividad y cohesión social. Con estos mismos objetivos, la Diputación Foral de Bizkaia se ha unido al proyecto del índice y se ha fijado como prioridad conocer la situación de envejecimiento de su población mayor de cara al desarrollo de políticas que mejoren su calidad de vida, a la vez que contribuyan al progreso y bienestar social de toda la población.

Los resultados que se presentan en este artículo muestran una buena situación general del envejecimiento activo de la población mayor de Bizkaia, por encima de la media europea. Sin embargo, la situación varía en función de la dimensión del índice que observemos, lo cual permite identificar las fortalezas y debilidades del envejecimiento activo actualmente en Bizkaia. Así, por un lado, en lo que respecta a la contribución de la población mayor a través del empleo, los resultados muestran que el trabajo remunerado en la población de Bizkaia mayor de 65 años es muy minoritario, con tasas de ocupación considerablemente más bajas que las de la gran mayoría de los países europeos.

Por otro lado, también destaca el bajo porcentaje de personas de 75 y más años que viven solas o en pareja, indicador que pretende recoger la autonomía de las personas mayores. Esta baja puntuación en nuestro contexto puede ser debida a una fuerte ideología familista y a un Estado de bienestar centrado en la familia, rasgo característico de los países del sur de Europa, en donde la mayor parte del cuidado (a personas mayores, niños/as y también a hijos/as mayores de edad) recae en la familia que comparte el mismo hogar. Otro indicador de esta misma dimensión, 'vida independiente y segura', en el que Bizkaia puntúa más bajo que la media europea y española es el relativo a la renta mediana disponible de la población mayor de 65 años respecto a aquellos menores de 65 años. Las pensiones de jubilación son fijadas por el Gobierno central de manera homogénea para el conjunto del Estado, lo que unido a unos salarios de la población trabajadora de Bizkaia superiores a la media española, puede provocar que la renta de las personas mayores sea, en comparación con las personas menores de 65 , más baja en Bizkaia que en el conjunto del Estado.

Por el contrario, los resultados también muestran que Bizkaia está a la cabeza de Europa en algunas áreas que favorecen el envejecimiento activo. Así, destaca la alta participación social de su población mayor, en el trabajo voluntario, el cuidado de personas, y actividades sindicales y políticas. Los datos de Bizkaia contrastan con el supuesto patrón europeo norte-sur que destacan algunos estudios sobre voluntariado, participación política o actividades políticas de protesta. Dicho patrón mostraría que son los países nórdicos y centroeuropeos los que tienen mayores tasas de participación, mientras que los países mediterráneos presentan los niveles más bajos (Hank y Erlinghagen, 2005; Neira, Portela y Vieira, 2010; Valencia, Cohen y Hermosilla, 2010). Por tanto, los buenos resultados de estos indicadores en el caso de Bizkaia nos estarían señalando un mayor parecido con los países nórdicos y del centro de Europa que con los países del sur.

Finalmente, Bizkaia obtiene una alta puntuación en la capacidad para envejecer activamente, en especial en los indicadores relacionados con la salud y la esperanza de vida, sensiblemente mejores que la media europea. Destaca también por el mayor contacto social de su población mayor, que además utiliza las nuevas tecnologías de la información y la comunicación en mayor proporción que la mayoría de los países europeos, lo que da cuenta de la importante capacidad que las personas mayores en Bizkaia para desarrollar un envejecimiento activo y saludable.

Una vez presentados los resultados del Índice de Envejecimiento Activo en Bizkaia, es preciso considerar algunas de las limitaciones conceptuales y metodológicas del índice. Si bien éste se creó como una herramienta para monitorizar el envejecimiento activo que tiene gran interés para aproximarnos a una foto comparada de la situación de los distintos países y regiones, el concepto de envejecimiento activo que subyace a él tiene un enfoque basado en la aportación económica y social que la población mayor puede hacer al desarrollo de las sociedades. Desde esta perspectiva, no refleja el bienestar de la población mayor y la mejora de su calidad de vida, sino el aprovechamiento del potencial de este grupo social como agente activo de la sociedad, y el interés en promover políticas que contribuyan a él. De 
hecho, son los indicadores relativos al empleo y a la participación social, es decir, al trabajo remunerado y no remunerado, los que mayor peso tienen dentro del índice, llegando a suponer el 54,3\% del total de la puntuación. En cambio, otros aspectos fundamentales para el envejecimiento activo, como la salud o las condiciones de vida y del entorno, sólo suponen el $17 \%$ y $6 \%$, respectivamente.

Además, el envejecimiento activo ha sido definido a escala macro, que puede resultar poco sensible a las singularidades y características de cada región y a las necesidades que las poblaciones mayores de cada territorio tienen en torno a envejecer de forma activa y saludable. Por ello, para avanzar en el desarrollo de un concepto de envejecimiento activo integral y adaptado a cada contexto, resultaría conveniente incorporar aquellas cuestiones que son importantes para las propias personas mayores en su vivencia de la vejez. Ello requeriría explorar cuáles son las dimensiones y los aspectos concretos que el índice debería considerar de acuerdo con la vivencia que del envejecimiento activo tienen sus protagonistas. Así, la distribución del peso que los diferentes aspectos del envejecimiento activo tienen dentro del índice podría ser diferente en consonancia con las necesidades y prioridades locales, que deberían ser identificadas.

Por otro lado, el índice tiene algunas limitaciones metodológicas cuando se calcula a escala subestatal. En primer lugar, la información estadística disponible para este nivel de desagregación subregional presenta un tamaño de muestra y nivel de comparabilidad con el resto de los países insuficientes. A pesar de que Eustat ofrece datos para el conjunto del País Vasco, la mayoría de los indicadores para Bizkaia proceden de encuestas poblacionales, es decir, no dirigidas específicamente a la población mayor y, por tanto, con tamaños muestrales para la población de 55 y más años de Bizkaia que no permiten la desagregación por otras variables de interés, como el nivel de estudios o la clase social. Además, dichas encuestas proveen datos sobre indicadores que no coinciden exactamente con los empleados originariamente en el índice. En nuestro estudio, hemos optado por producir datos primarios ad hoc a partir de la Encuesta de Envejecimiento Activo en
Bizkaia, para el cálculo de aquellos indicadores en los que los datos secundarios tenían menor calidad o comparabilidad.

A pesar de las dificultades de obtener datos a escala subregional, lo cierto es que la mayoría de las competencias políticas relacionadas con el envejecimiento (incluyendo el envejecimiento activo) son desarrolladas a esa escala, lo cual crea un importante desajuste entre la falta de información estadística confiable y el nivel territorial en el que se implementan la mayoría de estas políticas. Por lo tanto, sería necesario promover progresivamente una mayor disponibilidad de datos estadísticos en la misma escala territorial en la que estas políticas se desarrollan, única forma de avanzar en una evaluación rigurosa de las políticas públicas y en una mayor adaptación de éstas a la ciudadanía. Por esta razón, sería altamente recomendable que, al menos en los Estados con un nivel alto de descentralización, como es el caso español, la Comisión Europea o Eurostat alentaran a los institutos regionales de estadística a ofrecer información comparable, como ya promueve en los institutos nacionales de estadística de cada país, en los que las operaciones estadísticas europeas son aplicadas directamente a muestras nacionales. Esta información estadística debería también ser representativa para áreas más pequeñas dentro de las regiones, y contar, además, con un tamaño muestral que permitiera realizar análisis en los diferentes subgrupos poblacionales.

Para concluir, el Índice de Envejecimiento Activo y los resultados que, de forma comparada a escala europea, se han presentado para Bizkaia suponen un paso en el reto de analizar la situación de envejecimiento de la población mayor y poner de manifiesto aquellos ámbitos en los que existen carencias para asegurar su bienestar y calidad de vida, así como la continuidad de su contribución al progreso social de las sociedades en las que viven. En todo caso, es necesario profundizar en un análisis que permita identificar desigualdades en el desarrollo de un envejecimiento activo y saludable de la población mayor, así como seguir monitorizando la situación a escala regional, de manera que sirva de apoyo a la implementación de políticas públicas en aquellos aspectos y subgrupos poblacionales con mayores carencias. 
Anexo. Fuente de datos, año y tamaño muestral para cada indicador del Îndice de Envejecimiento Activo

\begin{tabular}{|c|c|c|c|c|}
\hline Dimensión & Indicador & Fuente de datos & Años & $\begin{array}{l}\text { Tamaño } \\
\text { muestral }\end{array}$ \\
\hline \multirow{4}{*}{ 1. Empleo } & 1.1. Tasa de ocupación en población de 55 a 59 años & \multirow{4}{*}{$\begin{array}{l}\text { Encuesta de Población en } \\
\text { Relación con la Actividad } \\
\text { del País Vasco (Eustat) }\end{array}$} & \multirow{4}{*}{$2012-2013$} & \multirow{4}{*}{$n=531$} \\
\hline & 1.2. Tasa de ocupación en población de 60 a 64 años & & & \\
\hline & 1.3. Tasa de ocupación en población de 65 a 69 años & & & \\
\hline & 1.4. Tasa de ocupación en población de 70 a 74 años & & & \\
\hline \multirow{4}{*}{$\begin{array}{l}\text { 2. Participación } \\
\text { social }\end{array}$} & $\begin{array}{l}\text { 2.1. Porcentaje de población } \geq 55 \text { años que realiza trabajo } \\
\text { voluntario en asociaciones }\end{array}$ & \multirow{4}{*}{$\begin{array}{l}\text { Encuesta de } \\
\text { Envejecimiento Activo en } \\
\text { Bizkaia (Diputación Foral } \\
\text { de Bizkaia) }\end{array}$} & \multirow{4}{*}{2014} & \multirow{4}{*}{$n=1.362$} \\
\hline & $\begin{array}{l}\text { 2.2. Porcentaje de población } \geq 55 \text { años que cuida a hijos/as o } \\
\text { nietos/as al menos } 1 \text { vez/semana }\end{array}$ & & & \\
\hline & $\begin{array}{l}\text { 2.3. Porcentaje de población } \geq 55 \text { años que cuida a personas } \\
\text { mayores o discapacitadas al menos } 1 \text { vez/semana }\end{array}$ & & & \\
\hline & $\begin{array}{l}\text { 2.4. Porcentaje de población } \geq 55 \text { años que participa en las } \\
\text { actividades de un sindicato, partido político u otro grupo con } \\
\text { actividad política }\end{array}$ & & & \\
\hline \multirow{8}{*}{$\begin{array}{l}\text { 3. Vida } \\
\text { independiente, } \\
\text { saludable y } \\
\text { segura }\end{array}$} & $\begin{array}{l}\text { 3.1. Porcentaje de población } \geq 55 \text { años que realiza actividad } \\
\text { física o deporte al menos } 5 \text { veces/semana }\end{array}$ & \multirow{2}{*}{$\begin{array}{l}\text { Encuesta de } \\
\text { Envejecimiento Activo en } \\
\text { Bizkaia (Diputación Foral } \\
\text { de Bizkaia) }\end{array}$} & \multirow[b]{2}{*}{2014} & \multirow[b]{2}{*}{$n=1.362$} \\
\hline & $\begin{array}{l}\text { 3.2. Porcentaje de población } \geq 55 \text { años que afirma no haber } \\
\text { tenido necesidades médicas o dentales no cubiertas (examen } \\
\text { o tratamiento) en los últimos } 12 \text { meses }\end{array}$ & & & \\
\hline & $\begin{array}{l}\text { 3.3. Porcentaje de población } \geq 75 \text { años que vive en una } \\
\text { vivienda unipersonal o en pareja }\end{array}$ & $\begin{array}{l}\text { Encuesta Demográfica del } \\
\text { País Vasco (Eustat) }\end{array}$ & 2011 & \\
\hline & $\begin{array}{l}\text { 3.4. Ratio entre la renta mediana disponible equivalente de la } \\
\text { población } \geq 65 \text { años y la de la población }<65 \text { años }\end{array}$ & \multirow{2}{*}{$\begin{array}{l}\text { Encuesta de Pobreza y } \\
\text { Desigualdades Sociales del } \\
\text { País Vasco (Departamento } \\
\text { de Empleo y Políticas } \\
\text { Sociales del Gobierno } \\
\text { Vasco, y Eustat) }\end{array}$} & \multirow{2}{*}{2012} & \multirow{2}{*}{$n=616$} \\
\hline & $\begin{array}{l}\text { 3.5. Porcentaje de población } \geq 65 \text { años que no se encuentra } \\
\text { en riesgo de pobreza }\end{array}$ & & & \\
\hline & $\begin{array}{l}\text { 3.6. Porcentaje de población } \geq 65 \text { años sin privación material } \\
\text { severa }\end{array}$ & \multirow{3}{*}{$\begin{array}{l}\text { Encuesta de } \\
\text { Envejecimiento Activo en } \\
\text { Bizkaia (Diputación Foral } \\
\text { de Bizkaia) }\end{array}$} & \multirow{3}{*}{2014} & \multirow{3}{*}{$n=1.362$} \\
\hline & $\begin{array}{l}\text { 3.7. Porcentaje de población } \geq 55 \text { años que no está } \\
\text { preocupada por ser víctima de un delito violento }\end{array}$ & & & \\
\hline & $\begin{array}{l}\text { 3.8. Porcentaje de población de } 55 \text { a } 74 \text { años que afirma } \\
\text { haber recibido algún tipo de formación el mes anterior }\end{array}$ & & & \\
\hline \multirow{6}{*}{$\begin{array}{l}\text { 4. Capacidad } \\
\text { y entornos } \\
\text { apropiados para } \\
\text { un envejecimiento } \\
\text { activo }\end{array}$} & $\begin{array}{l}\text { 4.1. Esperanza de vida a los } 55 \text { años dividida entre } 50 \text {, para } \\
\text { calcular la proporción de esperanza de vida alcanzada sobre } \\
\text { el objetivo de } 105 \text { años }\end{array}$ & $\begin{array}{l}\text { Estadística de Mortalidad } \\
\text { del País Vasco (Eustat) }\end{array}$ & 2012 & $\begin{array}{l}\text { Registro de } \\
\text { población }\end{array}$ \\
\hline & $\begin{array}{l}\text { 4.2. Porcentaje de esperanza de salud sobre la esperanza de } \\
\text { vida total a los } 55 \text { años }\end{array}$ & $\begin{array}{l}\text { Encuesta de Salud del País } \\
\text { Vasco (Departamento de } \\
\text { Empleo y Políticas Sociales } \\
\text { del Gobierno Vasco, y } \\
\text { Eustat) }\end{array}$ & 2013 & $n=1.801$ \\
\hline & 4.3. Salud mental en población $\geq 55$ años & \multirow{3}{*}{$\begin{array}{l}\text { Encuesta de } \\
\text { Envejecimiento Activo en } \\
\text { Bizkaia (Diputación Foral } \\
\text { de Bizkaia) }\end{array}$} & \multirow{3}{*}{2014} & \multirow{3}{*}{$n=1.362$} \\
\hline & $\begin{array}{l}\text { 4.4. Porcentaje de población de 55-74 años que utiliza } \\
\text { internet al menos } 1 \text { vez/semana }\end{array}$ & & & \\
\hline & $\begin{array}{l}\text { 4.5. Porcentaje de población } \geq 55 \text { años con contacto diario o } \\
\text { frecuente con amistades, familiares o colegas }\end{array}$ & & & \\
\hline & $\begin{array}{l}\text { 4.6. Porcentaje de población de } 55 \text { a74 años con educación } \\
\text { secundaria superior o terciaria }\end{array}$ & $\begin{array}{l}\text { Encuesta de Población en } \\
\text { Relación con la Actividad } \\
\text { del País Vasco (Eustat) }\end{array}$ & 2012-2013 & $n=1.345$ \\
\hline
\end{tabular}


COMISIÓN EUROPEA (2015): The 2015 Ageing Report. Economic and Budgetary Projections for the 28 EU Member States (2013-2060), serie European Economy, $\mathrm{n}^{0}$ 3/2015, Bruselas, Oficina de Publicaciones de la Unión Europea [<http:// ec.europa.eu/economy_finance/publications/ european_economy/2015/pdf/ee3_en.pdf〉].

- (2013): Communication from the Commission to the European Parliament, the Council, the European Economic and Social Committee and the Committee of the Regions: Towards Social Investment for Growth and Cohesion including implementing the European Social Fund 2014-2020, COM(2013) 83 final, Bruselas, Comisión Europea.

- (2010): Europa 2020. Una estrategia para un crecimiento inteligente, sostenible e integrador, $\mathrm{COM}$ (2010) 2020 final, Bruselas, Comisión Europea [rhttp://eur-lex.europa.eu/LexUriServ/ LexUriServ.do?uri=COM:2010:2020:FIN:ES:P $D F\rangle$.

CONSEJO DE LA UNIÓN EUROPEA (2012): Declaración del Consejo sobre el Año Europeo del Envejecimiento Activo y de la Solidaridad Intergeneracional (2012): estrategia futura, 17468/12, SOC 992, SAN 322, Bruselas, Consejo de la Unión Europea [rhttp://data. consilium.europa.eu/doc/document/ST-174682012-INIT/es/pdf)].

DIPUTACIÓN FORAL DE BIZKAIA (2013): Plan Estratégico de Personas Mayores de Bizkaia, Bilbao, Diputación Foral de Bizkaia [<http://www. bizkaia.net/home2/archivos/DPTO3/ Temas/PLAN\%20ESTRATEGICO $\% 20$ DE $\% 20$ PERSONAS\%2OMAYORES.pdf?idioma=CA>].

- (2010): Plan de Acción para la Promoción del Envejecimiento Activo en Bizkaia 2010-
2011, Bilbao, Diputación Foral de Bizkaia [<http://www.bizkaia.net/home2/Archivos/ DPTO3/Temas/Pdf/Plan\%2ode\%20 actuaci\%C3\%B3n\%2oen\%2omateria\%20 de\%2oenvejecimiento\%2oactivo.pdf $\rangle$.

- (2006): Plan Gerontológico de Bizkaia 2006-2011, Bilbao, Diputación Foral de Bizkaia.

EUSTAT (2014a): Estadística Municipal de Habitantes, Vitoria-Gasteiz, Eustat-Instituto Vasco de Estadística [<http://www.eustat.eus/ estadisticas/tema_159/opt_o/temas. html\#axzz3ngK8b7|O>].

- (2014b): Proyecciones de población, Vitoria-Gasteiz, Instituto Vasco de Estadística [rhttp://www. eustat.eus/estadisticas/tema_163/opt_o/ temas.html\#axzzznlukugAh〉].

GOBIERNO VASCO (2015): Estrategia Vasca de Envejecimiento Activo 2015-2020, VitoriaGasteiz, Eusko Jaurlaritza-Gobierno Vasco [«http://siis.net/es/documentacion/catalogo/ Record/503054>].

HANK, K.; y ERLINGHAGEN, M. (2010): "Dynamics of volunteering in older Europeans", The Gerontologist, vol. 50, nํㅜ 2, págs. 170-178 [〈http://doi.org/10.1093/geront/gnp122〉].

KALACHE, A.; y KICKBUSCH, I. (1997): “A global strategy for healthy ageing”, World Health, vol. 50, $\mathrm{n} \stackrel{-}{-} 4$, págs. 4-5.

LUXÁN, M.; y MARTíN, U. (2012): “Demografía”, en EUSTAT, Informe socioeconómico de la C.A. de Euskadi 2012, Vitoria-Gasteiz, Eustat-Instituto Vasco de Estadística, págs. 1-46 [<http://www.eustat. eus/elementos/eleooog80o/ti_informesocioeconomico-de-la-ca-de-euskadi-2012demografia-pdf-534-kb/infooog880_c.pdf`]. 
NEIRA, I.; PORTELA, M.; y VIEIRA, E. (2010): “Social capital and growth in European Regions", Regional and Sectoral Economic Studies, vol. 10, ํㅡ 2, págs. 19-28.

OMS (2002): Active Ageing. A Policy Framework, Ginebra, Organización Mundial de la Salud.

PÉREZ DÍAZ, J. (2006): Demografía y envejecimiento. Lecciones de gerontología I, serie Informes Portal Mayores, $\mathrm{n}^{\circ}$ 51, Madrid, Portal Mayores [〈http://envejecimiento.csic.es/documentos/ documentos/perez-demografia-01.pdf $>$.

SEN, A. K. (2009): The Idea of Justice, Penguin, Londres.

- (1993): "Capability and well-being”, en NUSSBAUM, M.; y SEN, A. K. (eds.), The Quality of Life, Oxford, Oxford University Press.
UNECE (2012): 2012 Vienna Ministerial Declaration. Ensuring a Society for All Ages: Promoting Quality of Life and Active Ageing, Viena, Comisión Económica para Europa de Naciones Unidas [<http:// www.unece.org/fileadmin/DAM/pau/age/ Ministerial_Conference_Vienna/Documents/

ECE.AC.30-2012-3.E.pdf)].

VALENCIA, J. F.; COHEN, E. H.; y HERMOSILLA, D. (2010): "Social trust and political protest. The mediating role of the value of power distance", Psicología Política, no 40, págs. 61-80.

ZAIDI, A. et al. (2013): Active Ageing Index 2012. Concept, Methodology and Final Results, Viena, European Centre [shttp://www1. unece.org/stat/platform/display/AAI/ VI.+Documentation>]. 